\title{
Fast Sodium Channel Gating Supports Localized and Efficient Axonal Action Potential Initiation
}

\author{
Christoph Schmidt-Hieber ${ }^{1,2}$ and Josef Bischofberger ${ }^{2,3}$ \\ ${ }^{1}$ Wolfson Institute for Biomedical Research, University College London, London, WC1E 6BT, United Kingdom, ${ }^{2}$ Physiologisches Institut der Universität \\ Freiburg, Abteilung I, D-79108 Freiburg, Germany, and ${ }^{3}$ Department of Biomedicine, Physiological Institute, University of Basel, CH-4056 Basel, \\ Switzerland
}

Action potentials (APs) are initiated in the proximal axon of most neurons. In myelinated axons, a 50-times higher sodium channel density in the initial segment compared to the soma may account for this phenomenon. However, little is known about sodium channel density and gating in proximal unmyelinated axons. To study the mechanisms underlying AP initiation in unmyelinated hippocampal mossy fibers of adult mice, we recorded sodium currents in axonal and somatic membrane patches. We demonstrate that sodium channel density in the proximal axon is $\sim 5$ times higher than in the soma. Furthermore, sodium channel activation and inactivation are $\sim 2$ times faster. Modeling revealed that the fast activation localized the initiation site to the proximal axon even upon strong synaptic stimulation, while fast inactivation contributed to energy-efficient membrane charging during APs. Thus, sodium channel gating and density in unmyelinated mossy fiber axons appear to be specialized for robust AP initiation and propagation with minimal current flow.

\section{Introduction}

Action potential (AP) initiation is an elementary step in neuronal signal processing. The temporal and spatial pattern of AP propagation along the axon and within the dendritic tree critically depends on the location where the AP is triggered. In the mammalian CNS, APs are preferentially initiated in the proximal axon (Stuart et al., 1997). Computational modeling, sodium $\left(\mathrm{Na}^{+}\right)$ imaging studies and $\mathrm{Na}^{+}$current recordings from axonal membrane patches suggest that a 40 - to 50 -times higher density of functionally specialized voltage-gated $\mathrm{Na}^{+}$(Nav) channels in the axon initial segment of neocortical pyramidal cells might be critically important for this phenomenon (Kole et al., 2008; Hu et al., 2009). In contrast to the large amount of data that has recently been published on Nav channel gating and density in the initial segment of myelinated axons, only very little is known about $\mathrm{Nav}$ channels in mammalian nonmyelinated axons.

In myelinated axons, APs propagate mainly passively along internodal sections. Since the specific capacitance of the myelinated membrane is very low, little energy is required to depolarize the membrane during an AP. By contrast, AP propagation in nonmyelinated axons depends largely on continuous ion flow across the axonal membrane. Since the ion gradient between extracellular space and cytoplasm needs to be maintained by the

Received Dec. 22, 2009; revised June 8, 2010; accepted June 18, 2010.

C.S.-H. is a Feodor Lynen scholar of the Alexander von Humboldt Foundation. This work was supported by grants from the Deutsche Forschungsgemeinschaft (SFB 780/B3 and Bi 642/2) and the Gatsby Charitable Foundation. We thank Peter Jonas for his continuous and generous support, helpful suggestions, and comments on this manuscript. We thank Henrik Alle, Boris Gutkin, Marco Martina, and Greg Stuart for their helpful comments on previous versions of this manuscript. We thank S. Becherer, M. Northemann, and K. Winterhalter for technical assistance.

Correspondence should be addressed to Christoph Schmidt-Hieber, Wolfson Institute for Biomedical Research, University College London, London, WC1E6BT, UK. E-mail: c.schmidt-hieber@ucl.ac.uk.

DOI:10.1523/JNEUROSCI.6335-09.2010

Copyright $\odot 2010$ the authors $\quad 0270-6474 / 10 / 3010233-10 \$ 15.00 / 0$
$\mathrm{Na}^{+}-\mathrm{K}^{+}$-ATPase, this process consumes considerably more energy than propagation in myelinated axons, and consequently, AP propagation in nonmyelinated axons of the gray matter has been assumed to be responsible for most of the energy consumption in the brain (Attwell and Laughlin, 2001; Attwell and Gibb, 2005). This view has recently been challenged by $\mathrm{Na}^{+}$and $\mathrm{K}^{+}$ current recordings evoked by AP waveforms in presynaptic hippocampal mossy fiber boutons (MFBs), suggesting that there is much less overlap of $\mathrm{Na}^{+}$and $\mathrm{K}^{+}$currents during the AP than previously thought (Alle et al., 2009). However, it is unclear how the kinetics of Nav channel gating contributes to this phenomenon. This question might be of particular relevance at the AP initiation site in the proximal axon, because this is where the highest Nav channel density can be found within the axon (Kress et al., 2008) and therefore, the largest amount of $\mathrm{Na}^{+}$ions per length is expected to flow at this location.

In the thin nonmyelinated axons of hippocampal granule cells, the so-called mossy fibers, immunohistochemical data (Kress et al., 2008, 2010) and axonal voltage recordings (SchmidtHieber et al., 2008) suggest high Nav channel densities. However, no direct functional analysis of these channels has been performed yet. To address this question, we analyzed the functional properties of Nav channels in outside-out patches from somata and axons of hippocampal granule cells. We found that on average, Nav channel density in the proximal axon is $\sim 5$ times higher than in the soma. Most importantly, the functional properties of somatic and axonal Nav channels appear to be different with up to 2-times faster activation and inactivation kinetics in the axon. Compartmental cable modeling revealed that fast activation kinetics was required to provide a robust axonal initiation site with a moderately enhanced density of Nav channels, while fast axonal inactivation kinetics allowed energy-efficient initiation of APs. 


\section{Materials and Methods}

Slice preparation. Transverse $350-\mu \mathrm{m}$-thick slices were cut from the hippocampus of 2- to 4-month-old C57BL/6 male or female mice using a custom-made vibratome (Geiger et al., 2002). Animals were kept in an oxygenated chamber for $10 \mathrm{~min}$, anesthetized with isoflurane (Forene, Abbott; $4-5 \%$ added to the inspiration air flow) and subsequently killed by decapitation, in accordance with national and institutional guidelines. Experiments were approved by the Animal Care Committee Freiburg (registry X-07/08A). For the dissection and the storage of the slices, a solution containing $87 \mathrm{~mm} \mathrm{NaCl}, 25 \mathrm{~mm} \mathrm{NaHCO}, 2.5$ $\mathrm{mm} \mathrm{KCl}, 1.25 \mathrm{~mm} \mathrm{NaH}_{2} \mathrm{PO}_{4}, 25 \mathrm{~mm}$ glucose, 75 mM sucrose, $7 \mathrm{~mm} \mathrm{MgCl}_{2}$, and $0.5 \mathrm{mM} \mathrm{CaCl}_{2}$ (equilibrated with $95 \% \mathrm{O}_{2} / 5 \% \mathrm{CO}_{2}$ ) was used. Slices were incubated at $35^{\circ} \mathrm{C}$ for $\sim 30 \mathrm{~min}$ and subsequently stored at room temperature.

Electrophysiology. Slices were superfused with a physiological extracellular solution containing $125 \mathrm{~mm} \mathrm{NaCl}, 25 \mathrm{~mm} \mathrm{NaHCO}_{3}, 2.5 \mathrm{~mm}$ $\mathrm{KCl}, 1.25 \mathrm{~mm} \mathrm{NaH}_{2} \mathrm{PO}_{4}, 25 \mathrm{~mm}$ glucose, $2 \mathrm{~mm}$ $\mathrm{CaCl}_{2}$, and $1 \mathrm{mM} \mathrm{MgCl}_{2}$. Mature granule cells and terminal axonal expansions resulting from the slicing procedure (bleb diameter: $\sim 2-3$ $\mu \mathrm{m})$ were identified using infrared differential interference contrast (IR-DIC) video microscopy (Shu et al., 2006). Whole-cell patchclamp recordings from axonal blebs were established under IR-DIC control using a pipette solution containing $130 \mathrm{~mm} \mathrm{CsCl}, 4 \mathrm{~mm}$ $\mathrm{MgCl}_{2}, 4 \mathrm{~mm} \mathrm{Na} \mathrm{N}_{2} \mathrm{ATP}, 10 \mathrm{~mm} \mathrm{Na}$-phosphocreatine, 10 mM EGTA, 10 mM HEPES, and 100 $\mu \mathrm{M}$ AlexaFluor 594 (Invitrogen; $\mathrm{pH}$ adjusted to

7.2 with $\mathrm{CsOH}$ ). Subsequently, the fluorescently labeled soma was identified using a CCD camera (EBFT 512; Princeton Instruments). The excitation light source (Polychrome II; T.I.L.L. Photonics) was coupled to the epifluorescent port of the microscope [Axioskop FS2 (Zeiss); 60× waterimmersion objective (Olympus)] via a light guide. To minimize bleaching and avoid phototoxicity, the intensity of the excitation light $(550 \mathrm{~nm})$ was reduced to $10 \%$ of the maximal value provided by the excitation light source. Patch pipettes (6-10 M $\Omega$ for somatic outside-out patch recordings, 6-15 $\mathrm{M} \Omega$ for axonal outside-out patch recordings, 3-4 $\mathrm{M} \Omega$ for nucleated patch recordings) were pulled from thick-walled borosilicate glass (outer diameter, $2.0 \mathrm{~mm}$; inner diameter, $0.6 \mathrm{~mm})$. For estimation of $\mathrm{Na}^{+}$current density, pipettes with similar tip resistances (7-9 M $\Omega$ ) were used for somatic and axonal recordings. Series resistance in whole-cell mode before withdrawal of the pipette was 20-40 M $\Omega$ for outside-out patch recordings and 8-15 $\mathrm{M} \Omega$ for nucleated patch recordings. Currents were measured with an Axopatch $200 \mathrm{~A}$ amplifier (Molecular Devices), filtered at $10 \mathrm{kHz}$ (internal 4-pole lowpass Bessel filter), and digitized at $50-100 \mathrm{kHz}$ using a CED 1401plus or Power1401 interface (Cambridge Electronic Design). Voltage command pulses were generated using a home-made data acquisition software (FPulse, U. Fröbe, Physiologisches Institut der Universität Freiburg, Freiburg, Germany) running under Igor 5 (WaveMetrics). To isolate $\mathrm{Na}^{+}$currents pharmacologically, $20 \mathrm{~mm}$ tetraethylammonium chloride (TEA), $3 \mathrm{~mm}$ 4-aminopyridine (4-AP), and $100 \mu \mathrm{M} \mathrm{CdCl}_{2}$ were added to the bath solution. Capacitive and leakage currents were subtracted using the pipette capacitance compensation circuit of the amplifier and two $\mathrm{P} / 4$ pulse sequences within each protocol. Patches were held at $-80 \mathrm{mV}$ throughout the experiment. Chemicals were obtained from Sigma, Merck, or Gerbu. Recordings were made at $23 \pm 2^{\circ} \mathrm{C}$. At this temperature, the fastest activation and deactivation time constants were $\sim 20 \mu \mathrm{s}$, close to the temporal resolution limit of the recording system.

To estimate the surface area of outside-out patches, capacitive current transients were elicited by test pulses from -60 to $-110 \mathrm{mV}$. Pipettes
B

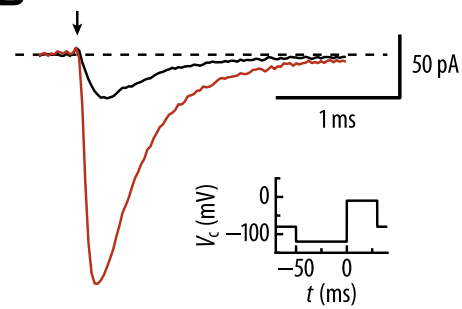

E
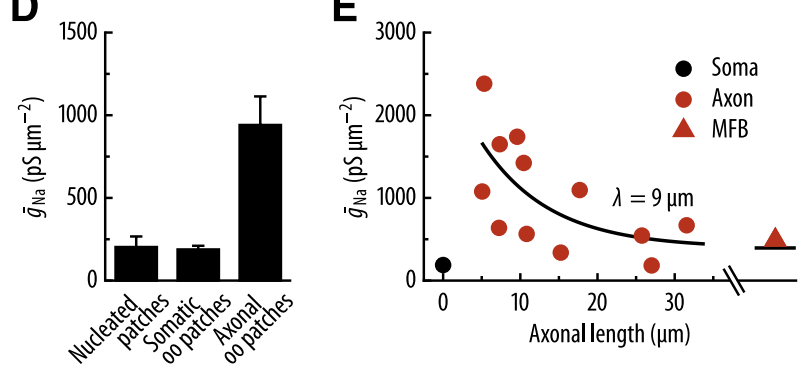

Figure 1. High density of Nav channels in the proximal mossy fiber axon. $\boldsymbol{A}$, Paired current-clamp recording from a granule cell soma and an axonal bleb showing an AP (upper traces) evoked by brief somatic current injection (lower trace). The AP was always iated in the axon. Data are from Schmidt-Hieber et al. (2008). The inset shows a scheme of the recording configuration. $\boldsymbol{B}, \mathrm{Na}^{+}$ 位e tip against a Sylgard microsphere (top black trace: somatic recording; bottom red trace: axonal recording). pest and axonal outside-out patches $(n=14)$. Axonal and somatic outside-out patches are from a paired dataset. $\boldsymbol{E}$, Plot of peak $\mathrm{Na}^{+}$conductance density against distance of the axonal bleb from the soma. The black circle represents the average of 14 somatic recordings, whereas the red circles show individual axonal measurements. $\mathrm{Na}{ }^{+}$conductance density in MFBs (red triangle) was taken from Engel and Jonas (2005). Data points were fitted with a monoexponential function plus a constant (black curve).

were coated with Sylgard for this set of experiments (Sylgard 184, Dow Corning) and had resistances of 7-9 $\mathrm{M} \Omega$ for both somatic and axonal outside-out patches. Transients were first recorded from intact outsideout patches in close proximity to a Sylgard microsphere attached to a glass tool. Then, the pipette tip was pressed against the microsphere, resulting in complete sealing of the tip (Sakmann and Neher, 1995). Care was taken to maintain the immersion depth of the pipette in the bath during this procedure. Average traces of 50-100 sweeps obtained in the two conditions were subtracted and corrected for leak current. The capacitive charge transfer was obtained by integrating over the initial 100 $\mu$ s of the transient after pulse onset (Engel and Jonas, 2005). Membrane surface area $A\left(\sim 4 \mu \mathrm{m}^{2}\right)$ of outside-out patches was calculated from the capacitance $(\sim 40 \mathrm{fF})$ assuming a specific membrane capacitance of 1 $\mu \mathrm{F} \cdot \mathrm{cm}^{-2}$. Membrane surface area of a nucleated patch was calculated from its diameter on the IR-DIC image at the end of a recording. The cross-sectional area of the pipette tip was subtracted.

Data analysis. For kinetic analysis, current traces were left-shifted in time with respect to the pulse protocol by $60 \mu$ s to account for the temporal delay introduced by the amplifier circuit, including the lowpass four-pole Bessel filter $\left(f_{\mathrm{c}}=10 \mathrm{kHz}\right)$ and the analog-digital converter. This delay was determined from the latency between the onset of the test pulse and the time point of half-maximal rise of tail currents during the deactivation protocol (Bischofberger et al., 2002). The time course of Nav channel activation was fitted with an exponential function with delayed onset

$$
I(t)= \begin{cases}I_{0}\left(1-\mathrm{e}^{\frac{-(t-\delta)}{\tau_{a}}}\right) & \text { for } t \leq \delta, \\ 0 & \text { for } t<\delta,\end{cases}
$$

where $I_{0}$ is the amplitude, $\tau_{\mathrm{a}}$ the activation time constant, and $\delta$ the delay of Nav channel activation. The time course of deactivation was fitted with 
A

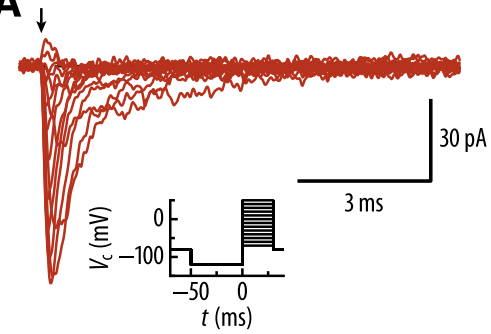

C

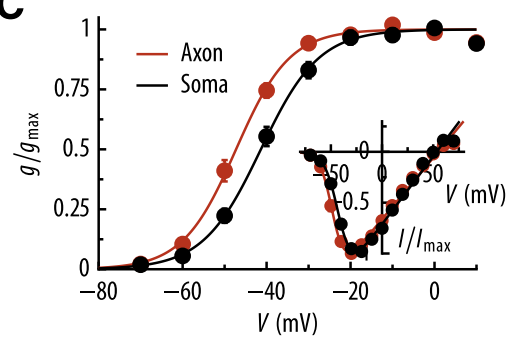

B

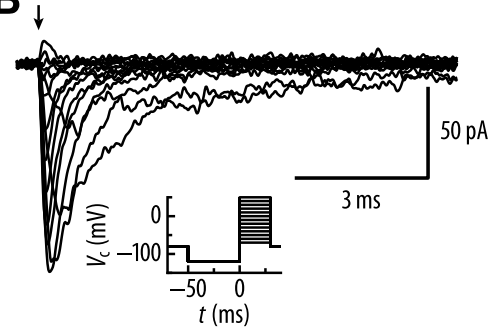

D

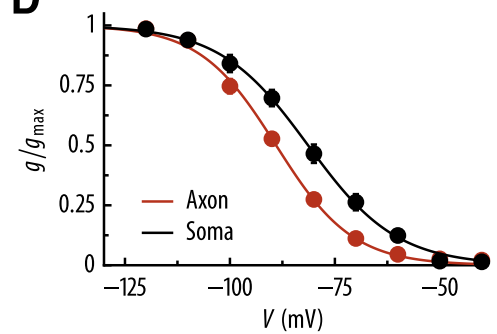

Figure 2. Axonal Nav channels activate and inactivate at more negative potentials than somatic channels. $\boldsymbol{A}, \boldsymbol{B}$, Traces show peak activation of $\mathrm{Na}^{+}$currents recorded from an axonal $(\boldsymbol{A})$ or a somatic $(\boldsymbol{B})$ outside-out patch of the same granule cell. The insets show the voltage pulse protocol. The arrows correspond to $t=0$ in the voltage pulse protocol. $\boldsymbol{C}, \boldsymbol{D}$, Peak activation $(\boldsymbol{C})$ and steady-state inactivation $(\boldsymbol{D})$ curves. Conductance was normalized to the maximal value and plotted against the test pulse potential. Data were fitted with Equation 2 ( $\boldsymbol{C}$ ) or 3 (D) (continuous curves). Midpoint potentials of activation ( $\boldsymbol{C}$ ) were $-47.3 \mathrm{mV}$ (slope factor $k=6.4 \mathrm{mV}$ ) in the axon (red) and $-41.4 \mathrm{mV}(k=6.9 \mathrm{mV})$ in the soma (black; $n=12)$. Midpoint potentials of inactivation (D) were $-89.0 \mathrm{mV}(k=9.1 \mathrm{mV})$ in the axon (red) and $-81.4 \mathrm{mV}(k=10.5 \mathrm{mV})$ in the soma (black). The inset in ( shows the mean peak current-voltage ( $I-V$ relation for somatic (black) and axonal (red) outside-out patches. The $I-V$ relation was fitted with the product of a linear and a Boltzmann function (Equation 4 ; continuous curves). Data in $C$ and $\boldsymbol{D}$ are from a paired dataset.

a single exponential. To account for inactivation at $V=-70 \mathrm{mV}$, a monoexponential function fitted to the late part of the trace was subtracted before analyzing the trace (Oxford, 1981; Engel and Jonas, 2005). Peak activation data were fitted with a Boltzmann function of the form

$$
f(V)=\frac{1}{1+\mathrm{e}^{\frac{V_{\text {mid }}-V}{k}}},
$$

where $V_{\text {mid }}$ is the midpoint potential and $k$ is the slope factor. Steadystate inactivation data were fitted with a Boltzmann function of the form

$$
g(V)=1-f(V)
$$

where $f(V)$ refers to Equation 2. To determine peak activation and steady-state inactivation, data were digitally filtered with a Gaussian filter $\left(f_{\mathrm{c}}=1.5 \mathrm{kHz}\right)$. This filter was not used when analyzing time constants. Gating properties are summarized in supplemental Table 1 (available at www.jneurosci.org as supplemental material).

Mean peak current-voltage $(I-V)$ relations were fitted with the product of a Boltzmann and a linear function

$$
I(V)=f(V)(s V+c),
$$

where $f(V)$ is the Boltzmann function of Equation 2, s is the slope, and $c$ is the $y$-intercept of the linear function. $\mathrm{Na}^{+}$reversal potential $E_{\mathrm{Na}}$ was calculated from the root of Equation 4 as $E_{\mathrm{Na}}=-c / s$. The peak $\mathrm{Na}^{+}$ conductance density $\bar{g}_{\mathrm{Na}}$ was calculated using a $30 \mathrm{~ms}$ test pulse to -10 $\mathrm{mV}$ preceded by a $50 \mathrm{~ms}$ prepulse to $-120 \mathrm{mV}$ to completely remove steady-state inactivation (see Fig. $2 C, D$ ). $\bar{g}_{\mathrm{Na}}$ was extrapolated by dividing current traces with an exponential function fitted to the decay of the current to account for the temporal overlap of activation and inactivation (supplemental Fig. $1 B$, available at www.jneurosci.org as supplemental material) (Keynes and Rojas, 1976). $\bar{g}_{\mathrm{Na}}$ was then calculated as $\bar{g}_{\mathrm{Na}}(V)=I_{\mathrm{Na}, \mathrm{c}} /(V A)$, where $I_{\mathrm{Na}, \mathrm{c}}$ is the corrected $\mathrm{Na}^{+}$peak current amplitude, $V$ is the test pulse potential, and $A$ is the membrane surface area of outside-out or nucleated patches, as appropriate. The axonal length was estimated from the shortest distance between the tip of the axonal recording pipette and the somatic point of origin of the axon using the IR-DIC images acquired during the experiments (Kole et al., 2007; Schmidt-Hieber et al., 2008). AP amplitudes were measured from the holding potential of $-80 \mathrm{mV}$. Voltage dependence of deactivation and activation time constants $\left(\tau_{\mathrm{d}}\right.$ and $\left.\tau_{\mathrm{a}}\right)$ in Figure $3 E$ were fitted with the function

$$
\tau(V)=\frac{p_{1}}{\cosh \left(\frac{V-p_{2}}{p_{3}}\right)}+p_{4},
$$

where $V$ is voltage and $p_{n}$ are free parameters.

AP latencies were measured as the difference in time at half-maximal amplitudes. Negative values denote APs preceding the somatic AP. The AP initiation site was determined as the site with the minimal AP latency.

Nonparametric Wilcoxon signed-rank or Mann-Whitney rank-sum tests were used to assess statistical significance. Values are given as mean \pm SEM. Error bars in the figures also indicate the SEM. Data were analyzed using C-Stimfit (C.S.-H.) and SciPy (http://www.scipy.org).

Modeling. Differential equations for kinetic Nav channel gating models were solved using the Q-matrix approach of Colquhoun and Hawkes (1977). Simulations of compartmental cable models were performed using NEURON 7 (Carnevale and Hines, 2006). In currentclamp simulations using compartmental cable models (see Figs. 5, $6 B, C$, 7; supplemental Figs. $5 B, 6,8$, available at www.jneurosci.org as supplemental material), voltage dependence of Nav channel gating rates was corrected for artificial shifts arising from Donnan and liquid junction potentials $(\sim 12 \mathrm{mV})$ and from the outside-out patch configuration ( $\sim 10 \mathrm{mV}$ for inactivation rates) (supplemental Fig. 3 and supplemental text, available at www.jneurosci.org as supplemental material). Experimental data and Nav channel gating models (see Figs. 1-4, 6A; supplemental Figs. 1-4, 5A, 7, available at www.jneurosci.org as supplemental material) are presented without correction for these shifts. Details of the modeling procedures are given in the supplemental material (available at www.jneurosci.org).

\section{Results}

A moderately higher density of Nav channels in the proximal mossy fiber axon

To directly measure the densities of Nav channels in hippocampal granule cells, we performed voltage-clamp recordings from somatic and axonal outside-out patches excised from granule cells and mossy fiber axons in acute hippocampal brain slices from adult mice. To record from similar membrane areas, two identical pipettes (range of resistances: 7-9 $M \Omega$ ) were used for recording of axonal and somatic $\mathrm{Na}^{+}$currents from the same granule cell (Fig. 1A, $B$, see Materials and Methods). Axonal recordings were obtained from artificial axonal endings at a distance of 5-35 $\mu \mathrm{m}$ from the soma. Using test pulses to $-10 \mathrm{mV}$, peak $\mathrm{Na}^{+}$current amplitudes were on average $\sim 5$ times larger in the axon than in the soma (Fig. $1 B$; supplemental Fig. $1 A$, available at www.jneurosci.org as supplemental material). To estimate the patch surface area, capacitance of outside-out patches was determined from capacitive currents evoked by $-50 \mathrm{mV}$ voltage steps (Fig. 1C) (Sakmann and Neher, 1995). Somatic and axonal outside-out patches had very similar capacitances ( $44 \pm 9 \mathrm{fF}, n=$ 5 , and $41 \pm 5 \mathrm{fF}, n=5$, respectively; $p>0.5$ ), corresponding to a patch area of $\sim 4 \mu \mathrm{m}^{2}$. To calculate peak $\mathrm{Na}^{+}$conductance den- 
A

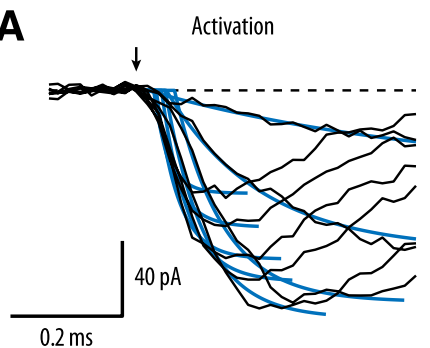

D

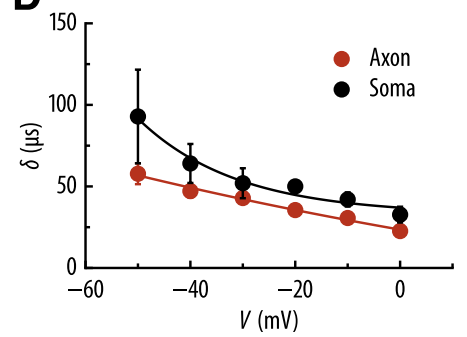

B

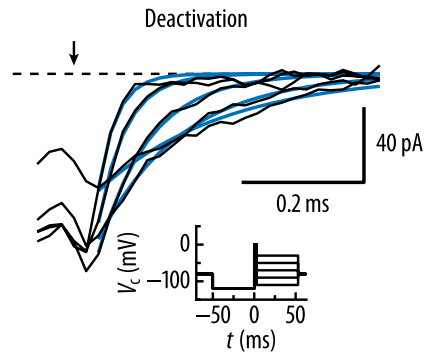

E

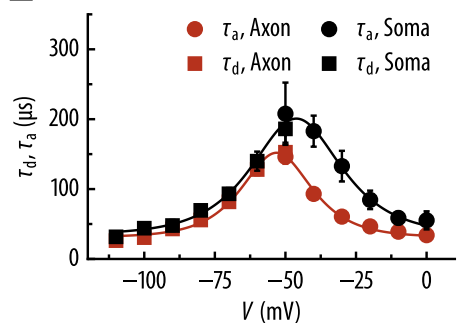

C

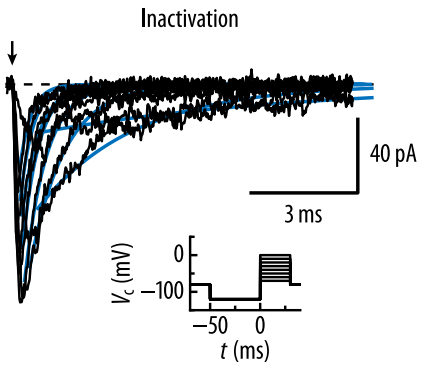

$\mathbf{F}$

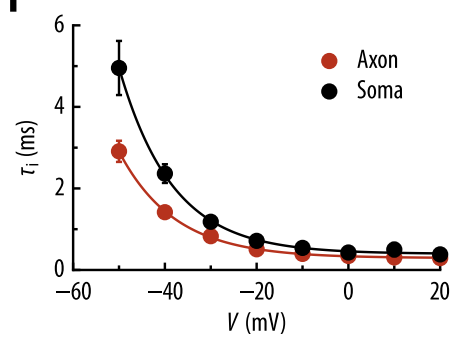

Figure 3. Axonal Nav channels activate and inactivate more rapidly than somatic channels. $A$, Time course of activation onset. The rising phase of the currents were fitted with monoexponential functions with delayed onset. Fit curves (blue) are shown superimposed to the current traces (black). The same voltage pulse protocol as in C (inset) was used. $\boldsymbol{B}$, Time course of deactivation. For test pulses $>-40 \mathrm{mV}$, monoexponential functions fitted to the late part of the current were subtracted before fitting monoexponential functions to the decay of the tail currents (blue curves; see Materials and Methods). $\boldsymbol{C}$, Time course of inactivation onset. The decay phase of the currents were fitted with monoexponential functions (blue curves). Insets in $\boldsymbol{B}$ and $\boldsymbol{C}$ show the voltage pulse protocols. The arrows in $\boldsymbol{A}-\boldsymbol{C}$ correspond to $t=0$ in the voltage pulse protocol. Dashed lines in $\boldsymbol{A}-\boldsymbol{C}$ are at $0 \mathrm{pA}$. $\boldsymbol{D}-\boldsymbol{F}$, Plot of activation delay $(\boldsymbol{D})$, activation and deactivation time constants $(\boldsymbol{E})$, and inactivation time constants $(\boldsymbol{F})$ against test pulse potential $(n=12)$. Data in $\boldsymbol{D}$ and $\boldsymbol{F}$ were fitted with exponential functions. Data in $\boldsymbol{E}$ were fitted with Equation 5 (see Materials and Methods). Data in $\boldsymbol{D}-\boldsymbol{F}$ are from a paired dataset.

A

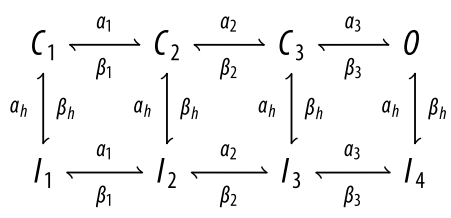

B

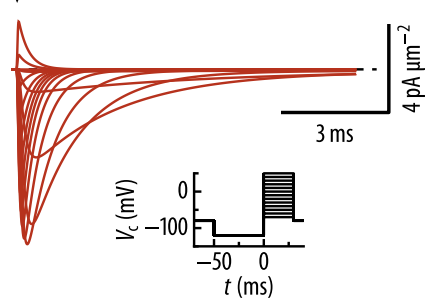

$\mathbf{E}$

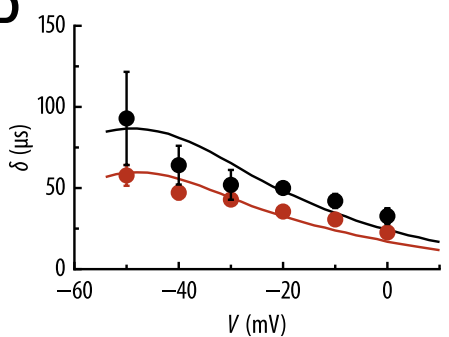

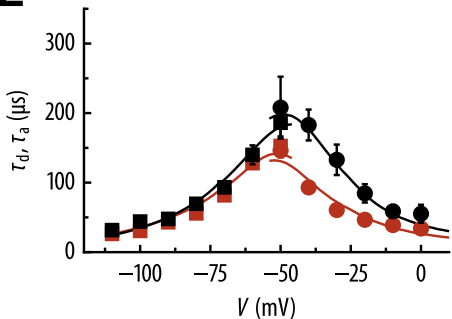

C

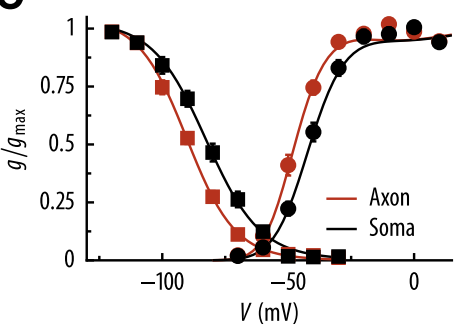

$\mathbf{F}$

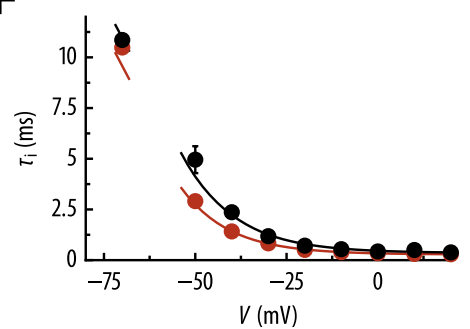

Figure 4. Gating kinetics can be reproduced with an eight-state kinetic gating model. $A$, Gating scheme of the eight-state gating model used for computational analysis of Nav channel gating. $B$, Simulated $\mathrm{Na}^{+}$currents using the eight-state gating model. Inset, Voltage pulse protocol. The arrow corresponds to $t=0$ in the voltage pulse protocol. C, Peak activation and steady-state inactivation curves. Continuous curves represent the results of the gating models that best fitted the data recorded from axon (red symbols) or soma (black symbols). $\mathbf{D}-\boldsymbol{F}$, Plot of activation delay $(\boldsymbol{D})$, deactivation and activation time constants $(\boldsymbol{E})$, and inactivation time constants $(\boldsymbol{F})$ against test pulse potentials. Curves, Best-fit gating models. Red symbols, Axonal data. Black symbols, Somatic data.

sities $\bar{g}_{\mathrm{Na}}$ from the peak $\mathrm{Na}^{+}$current amplitudes and the patch area, we divided current traces with an exponential function fitted to the decay of the current to account for the temporal overlap of activation and inactivation (supplemental Fig. $1 B$, available at www.jneurosci.org as supplemental material) (Keynes and Rojas, 1976). $\bar{g}_{\mathrm{Na}}$ was on average $188 \pm 22 \mathrm{pS} \cdot \mu \mathrm{m}^{-2}(18.8 \pm 2.2$ $\left.\mathrm{mS} \cdot \mathrm{cm}^{-2}\right)$ in the soma and $941 \pm 173 \mathrm{pS} \cdot \mu \mathrm{m}^{-2}$ in the axon measured at a distance of $5-35 \mu \mathrm{m}$ from the soma $(n=14$ paired recordings from granule cell soma and axon, respectively; $p<$ 0.001 ). As a second independent estimate of somatic Nav channel density, we performed nucleated-patch recordings (Martina and Jonas, 1997) revealing a conductance density of $203 \pm 64$ $\mathrm{pS} \cdot \mu \mathrm{m}^{-2}$, similar to the somatic outside-out patch conductance (Fig. $1 D)(n=6 ; p>0.9) \cdot \bar{g}_{\mathrm{Na}}$ was highest in the most proximal recordings at $\sim 5 \mu \mathrm{m}$ distance from the soma (Fig. $1 E)(\sim 1670$ $\left.\mathrm{pS} \cdot \mu \mathrm{m}^{-2}\right)$ and decreased toward more distal parts to an asymp- 
A
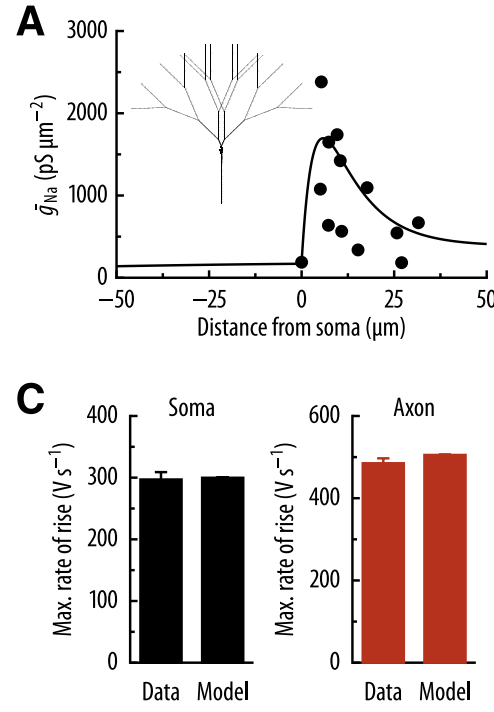

B

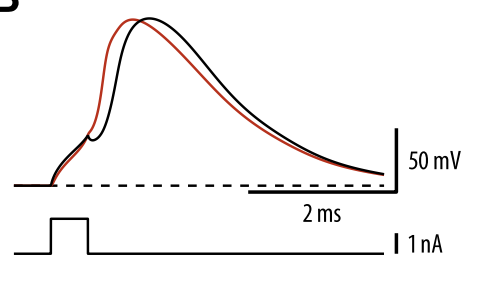

D

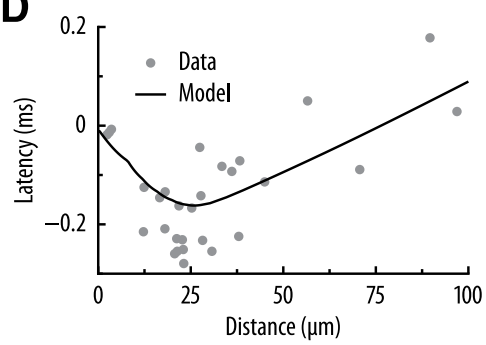

Figure 5. Axonal AP initiation in a detailed compartmental model using experimentally determined $\mathrm{Na}^{+}$conductance densities. $\boldsymbol{A}$, Plot of measured (black circles) and simulated (continuous curve) peak $\mathrm{Na}^{+}$conductance density against distance from soma. The inset shows a shape plot of the cell with NEURON. $\boldsymbol{B}$, Simulation of AP initiation and propagation within a granule cell with NEURON using the best-fit Nav channel gating model as well as experimentally determined Nav channel densities and kinetics. The AP was evoked by a brief current injection into the soma $(0.5 \mathrm{~ms}, 2 \mathrm{nA})$. Black trace, Somatic AP. Red trace, Axonal AP at a distance of $30 \mu \mathrm{m}$ from the soma. The dashed line is at $-80 \mathrm{mV}$. C, Bar graphs comparing experimentally determined AP properties (Schmidt-Hieber et al., 2008) to model results. The AP was evoked by brief current injection, as shown in $\boldsymbol{B}$. The axon of the compartmental model cell was cut at $30 \mu \mathrm{m}$ distance from the soma and a bleb ( $2 \mu \mathrm{m}$ diameter) was connected to the cut end. Left, Maximal rate of rise in the soma. Experiment, $297 \pm 12 \mathrm{~V} \cdot \mathrm{s}^{-1} ;$ model, $300 \mathrm{~V} \cdot \mathrm{s}^{-1}$. Right, Maximal rate of rise in the axon. Experiment, $485 \pm 12 \mathrm{~V} \cdot \mathrm{s}^{-1} ;$ model, $505 \mathrm{~V} \cdot \mathrm{s}^{-1}$. D, Plot of axonal AP latencies against distance from soma. The simulated AP was initiated in the axon at a distance of $\sim 30 \mu \mathrm{m}$ from the soma. The gray circles show experimentally determined values from 22 granule cells (Schmidt-Hieber et al., 2008).

totic value of $\sim 400 \mathrm{pS} \cdot \mu \mathrm{m}^{-2}$. Fitting a monoexponential function to the data points revealed that the channel density decayed with a length constant of $\sim 9 \mu \mathrm{m}$. A similar hot spot of Nav channels close to the axon hillock was found in hippocampal mossy fibers of juvenile rats using immunohistochemistry (Kress et al., 2008). Thus, peak Nav channel density at a hot spot close to the soma is 9 times the somatic density. However, with increasing distance from the soma, density decreases to an asymptotic value of about twice the somatic density, leading to an average density ratio of $\sim 5$ throughout the proximal $35 \mu \mathrm{m}$ of the axon. This is substantially less than the density ratio that has been estimated for the axon initial segment of neocortical and CA1 pyramidal cells ( $\sim 40-50$ times, Kole et al., 2008; Hu et al., 2009; Lorincz and Nusser, 2010).

\section{Axonal Nav channels activate and inactivate at more negative potentials than somatic channels}

It has previously been reported that axonal Nav channels of neocortical pyramidal cells activate and inactivate at more negative potentials than somatic channels (Colbert and Pan, 2002; Kole et al., 2008; Hu et al., 2009). To test whether a similar difference can be found in granule cell axons as well, we measured peak activation and steady-state inactivation of axonal and somatic Nav channels (Fig. 2). To increase the current amplitudes recorded from somatic patches, larger pipettes were used for somatic recordings during these experiments. Midpoint potentials $\left(V_{\text {mid }}\right)$ of the peak activation curve were $-46 \mathrm{mV}$ in the axon and -40 $\mathrm{mV}$ in the soma, corresponding to a voltage shift of about $-6 \mathrm{mV}$ for axonal Nav channels (Fig. 2A-C). Similarly, the midpoint potential of the steady-state inactivation curve in the axon (red) was shifted by $-8 \mathrm{mV}$ relative to the soma (black; Fig. 2D). In contrast to recent findings in layer $\mathrm{V}$ pyramidal cells ( $\mathrm{Hu}$ et al., 2009), we could not detect a gradual decrease of $V_{\text {mid }}$ of activation along the axon (supplemental Fig. 2, available at www.jneurosci.org as supplemental material). The low activation threshold of axonal Nav channels starting at the very beginning of the axon is consistent with recent immunohistochemical data revealing that the Nav 1.6 isoform is the dominant subunit in granule cell axon initial segments, while Nav 1.2 cannot be detected (Kress et al., 2010). In summary, both activation and inactivation occur at more negative potentials in proximal granule cell axons.

\section{Axonal Nav channels activate and inactivate faster than somatic channels} In addition to the voltage dependence, Nav channel function critically depends on gating kinetics. To examine differences in gating kinetics between the proximal axon and the soma of granule cells, we measured activation, deactivation, and inactivation time constants (Fig. 3). Activation time constants $\tau_{\mathrm{a}}$ were determined by fitting a monoexponential function with delayed onset to the rising phase of $\mathrm{Na}^{+}$ currents (Fig. 3A). The delay was necessary because currents activated with a sigmoidal onset that could not be accounted for by the recording circuit (see Materials and Methods). Deactivation time constants $\tau_{\mathrm{d}}$ were measured by fitting monoexponential functions to the decay of tail currents evoked by a brief $300 \mu$ s voltage pulse to 0 $\mathrm{mV}$, followed by test pulses to voltages between -110 and -50 $\mathrm{mV}$ (Fig. $3 B$ ). To account for the temporal overlap of deactivation and inactivation at voltages $\geq-70 \mathrm{mV}$, a monoexponential function was fitted to the late part of the current and subtracted before fitting the monoexponential function to the tail current (Oxford, 1981; Engel and Jonas, 2005). These measurements revealed that in the voltage range above the AP threshold of about $-50 \mathrm{mV}$ (Kress et al., 2008), activation was substantially faster in the axon (Fig. $3 E$ ). At a membrane potential of $-40 \mathrm{mV}$, the activation time constant was $93 \pm 7 \mu$ s in the axon and $183 \pm 22$ $\mu \mathrm{s}$ in the soma $(n=12 ; p<0.005)$ (supplemental Table 1 , available at www.jneurosci.org as supplemental material). Additionally, activation delay was significantly shorter in the axon at -20 and $0 \mathrm{mV}$ (Fig. 3D). By contrast, deactivation time constants did not significantly differ for voltages $>-100 \mathrm{mV}$ (Fig. $3 B, E$ ). Inactivation time constants $\tau_{\mathrm{i}}$ were obtained by fitting a monoexponential function to the decay of $\mathrm{Na}^{+}$currents at command voltages between -50 and $20 \mathrm{mV}$ (Fig. 3C). As with activation kinetics, inactivation was significantly faster in the axon than in the soma in a voltage range between -50 and $-10 \mathrm{mV}$ (Fig. $3 F$ ). In contrast to more depolarized membrane potentials, inactivation kinetics at subthreshold potentials was very similar (supplemental Fig. 4, available at www.jneurosci.org as supplemental material). Together, our data indicate that both activation and inactivation kinetics of $\mathrm{Nav}$ channel gating differs substantially in a voltage range of -50 to $-10 \mathrm{mV}$ corresponding to the early 
phase of action potentials, including onset and early upstroke. This suggests that functional properties of axonal Nav channels are specialized for efficient action potential initiation.

\section{Nav channel gating and AP initiation can be faithfully} reproduced using an eight-state Nav channel gating model

To test the functional consequences of the differences in Nav channel gating between the axon and the soma, we developed kinetic gating models of Nav channel gating (Fig. 4). We first tried to fit a standard Hodgkin-Huxley type gating model $(\mathrm{HH}$ model) to the experimental data using three identical gates for the activation and a single one for the inactivation process (Hodgkin and Huxley, 1952). However, the $\mathrm{HH}$ model failed to fit $\tau_{\mathrm{a}}$ and $\tau_{\mathrm{d}}$ at $-50 \mathrm{mV}$, which were very close to each other in the experimental data (supplemental Fig. 5, available at www.jneurosci.org as supplemental material), as observed previously for somatic and axonal Nav channels (Oxford, 1981; Baranauskas and Martina, 2006). To represent the $\mathrm{Na}^{+}$current data more precisely, we developed an eight-state gating model with different transition rates between multiple closed states, inactivated states, and a single open state (Fig. $4 A$; see supplemental material, available at www.jneurosci.org) (Vandenberg and Bezanilla, 1991; Baranauskas and Martina, 2006). In contrast to HH-type gating schemes, this model may account for the cooperative gating of different voltage-sensing domains of the Nav channel protein (Chanda et al., 2004). The model could well describe all experimental observations, including $\tau_{\mathrm{a}}$ and $\tau_{\mathrm{d}}$ at $-50 \mathrm{mV}$ (Fig. $4 B-F$ ).

We then inserted these $\mathrm{Na}^{+}$conductances into a compartmental cable model of a granule cell (Schmidt-Hieber et al., 2007), using an average channel density in the proximal axon of $940 \mathrm{pS} \cdot \mu \mathrm{m}^{-2}$ according to our measured data (Fig. $1 D, E$ ). Similarly, the spatial distribution of Nav channels was identical to the experimentally observed densities with an asymptotic value of $400 \mathrm{pS} \cdot \mu \mathrm{m}^{-2}$ for the distal axon (Fig. $5 A$ ). This compartmental model faithfully reproduced the granule cell AP phenotype, including maximal rate of rise and decay (Fig. $5 B, C$; supplemental Fig. 6, available at www.jneurosci.org as supplemental material). The AP was initiated at a distance of $\sim 30 \mu \mathrm{m}$ from the soma and propagated along the axon with a speed of $\sim 0.25 \mathrm{~m} \mathrm{~s}^{-1}$, similar to experimentally determined values (Fig. 5D; supplemental Fig. 6, available at www.jneurosci.org as supplemental material) (Kress et al., 2008; Schmidt-Hieber et al., 2008). Thus, the compartmental model faithfully reproduces experimental observations using low-intensity stimulation (Schmidt-Hieber et al., 2008).

\section{Fast activation kinetics provides a robust initiation site upon} weak and strong synaptic stimuli

Next, we tested how the compartmental model would perform when stronger excitatory synaptic stimuli were used to evoke APs. To assess the effects of activation kinetics under these conditions, we used two further axonal gating models with slower activation time constants (Fig. $6 \mathrm{~A}$ ): a model of Nav channel gating in MFBs in the distal granule cell axon (E\&J model) (Engel and Jonas, 2005) and a gating model that had previously been fitted to $\mathrm{Na}^{+}$current recordings from the soma and the axon of layer V pyramidal cells (M\&S model) (Mainen et al., 1995; Kole et al., 2008). We then distributed 500 synapses over the dendritic tree of a model cell and measured the AP initiation site while increasing the conductance of the synapses between simulation runs (Fig. 6B). When the experimentally observed Nav channel kinetics and densities ( $940 \mathrm{pS} \cdot \mu \mathrm{m}^{-2}$ in the proximal axon) were used, APs were initiated in the proximal axon throughout the
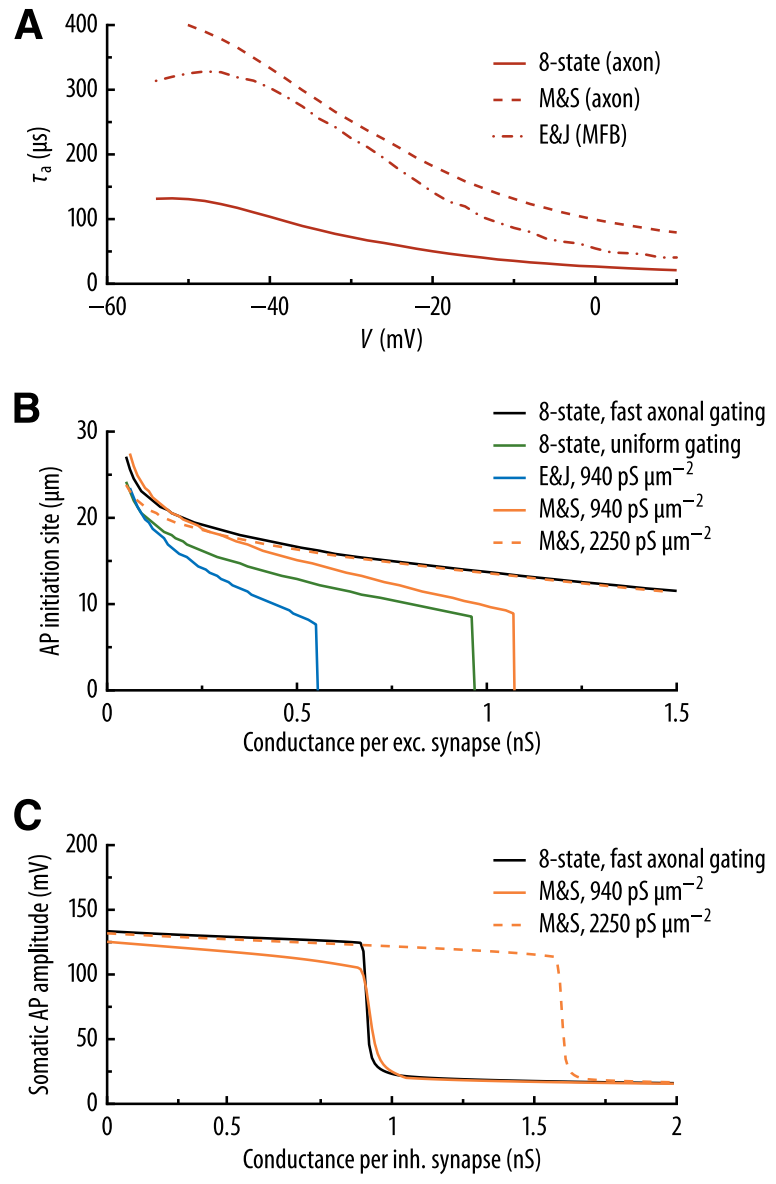

Figure 6. Fast activation kinetics enables AP initiation with relatively low axonal Nav channel density upon strong stimulation. $\boldsymbol{A}$, Plot of activation time constants against test pulse potentials. The continuous curve represents the eight-state gating model for the proximal axon as shown in Figure 4. The dashed curve represents a gating model developed for neocortical LV pyramidal cell axons (M\&S model) (Mainen et al., 1995; Kole et al., 2008). The dotted-dashed curve represents a model of Nav channel gating in MFBs in the distal granule cell axon (E\&J model) (Engel and Jonas, 2005). Note that activation time constants are considerably slower in the M\&S and E\& J models. $\boldsymbol{B}$, The AP initiation site (axonal distance from soma) was plotted against synaptic conductance (per single synapse of a total of 500) used to evoke the AP in a compartmental model. Positive distances denote axonal AP initiation. Black curve, The eight-state gating model was inserted with nonuniform kinetics and an average $\mathrm{Na}^{+}$conductance density of $940 \mathrm{ps} \cdot \mu \mathrm{m}^{-2}$ in the proximal axon. Green curve, The eight-state gating model was inserted with uniform kinetics and an average $\mathrm{Na}^{+}$conductance density of 940 $\mathrm{pS} \cdot \mu \mathrm{m}^{-2}$ in the proximal axon. Blue continuous curve, The E\&J model was inserted with an average $\mathrm{Na}^{+}$conductance density of $940 \mathrm{pS} \cdot \mu \mathrm{m}^{-2}$ in the proximal axon. Orange continuous curve, The M\&S model was inserted with an average $\mathrm{Na}^{+}$conductance density of $940 \mathrm{pS} \cdot \mu \mathrm{m}^{-2}$ in the proximal axon. Orange dashed curve, The M\&S model was inserted with an average $\mathrm{Na}^{+}$conductance density of $2250 \mathrm{pS} \cdot \mu \mathrm{m}^{-2}$ in the proximal axon. The sharp drops in the curves denote the conductance beyond which the initiation site swaps from an axonal location (positive distance) to a dendritic location (negative distance). When the E\&J or M\&S models with slower activation kinetics are used, axonal Nav channel density needs to be strongly enhanced to maintain the robustness of the initiation site upon strong synaptic input. By contrast, the combination of fast activation and nonuniform kinetics allows for a relatively low axonal Nav channel density. C, APs were evoked by near-threshold stimulation with excitatory synapses in a compartmental model. In addition, inhibitory synapses were distributed along the proximal $30 \mu \mathrm{m}$ of the axon to simulate axoaxonic inhibition. Somatic AP amplitude was plotted against conductance per inhibitory synapse. The same color code as in $\boldsymbol{B}$ was used. When the M\&S model with a high axonal $\mathrm{Na}^{+}$conductance density is used, inhibition needs to be 1.7 times stronger to prevent AP generation than in the eight-state model with a low density.

tested range of synaptic conductances from 0.1 to $1.5 \mathrm{nS}$ per synapse. By contrast, when the somatic channel kinetics with slower activation time constants was used uniformly throughout the cell, the initiation site swapped into the dendrites at $\sim 1 \mathrm{nS}$ per synapse (Fig. $6 \mathrm{~B}$, continuous green curve). 
A

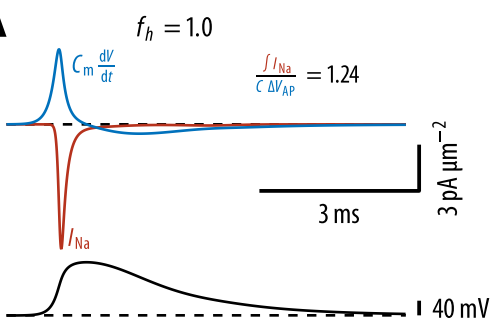

B

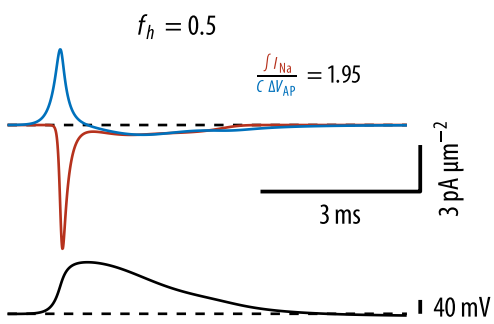

C $f_{h}=2.5$

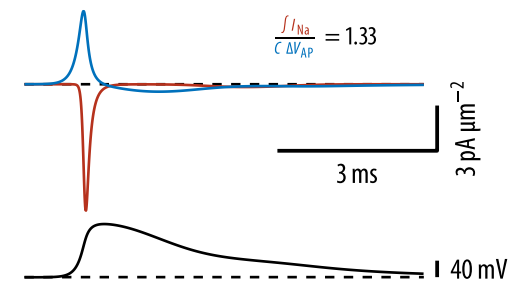

D

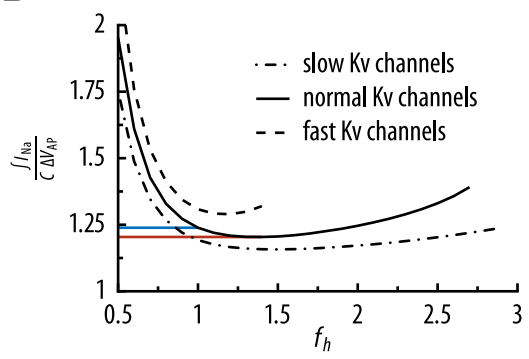

E

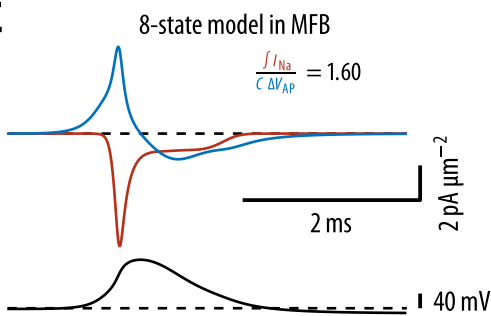

$\mathbf{F}$

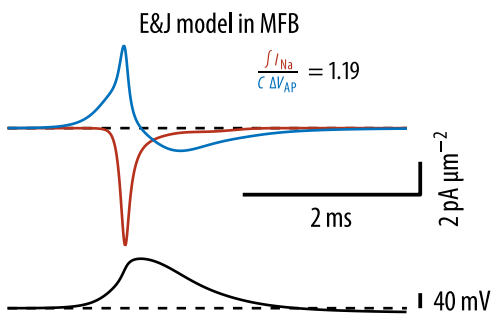

Figure 7. Axonal inactivation kinetics is tuned for energy-efficient AP initiation. A-C, APs were evoked by a brief current injection at one end of a very long cylinder ( $10 \mathrm{~mm}$ length, $1 \mu \mathrm{m}$ diameter). APs (black traces at bottom) and $\mathrm{Na}^{+}$(red traces) and capacitive (blue traces) currents were measured at the center of the cylinder. Energy efficiency was assessed as the ratio of total $\mathrm{Na}^{+}$charge transfer and the minimal charge that is required to depolarize the membrane during the AP. $\boldsymbol{A}$, The experimentally determined inactivation kinetics was used $\left(f_{h}=1\right)$. $\boldsymbol{B}$, Inactivation kinetics was slowed down by multiplying inactivation rates with the scaling factor $f_{h}=0.5$. C , Inactivation kinetics was accelerated by multiplying inactivation rates with $f_{h}=2.0 . \boldsymbol{D}$, Energy efficiency was plotted against the inactivation rates scaling factor $f_{h}$ (continuous black curve). The experimentally determined kinetics at $f_{h}=1$ yielded an energy efficiency of 1.24 (blue horizontal line), close to the minimum of 1.21 (red horizontal line). To assess the impact of Kv channel kinetics, simulations were repeated with slow Kv channel kinetics (activation gating rates multiplied with a factor of 0.5 , dotted-dashed curve) and with fast $\mathrm{Kv}$ channel kinetics (activation gating rates multiplied with a factor of 1.5, dashed curve), yielding similar results. $\boldsymbol{E}, \boldsymbol{F}$, Same simulations as in $\boldsymbol{A}-\boldsymbol{C}$ in a thinner cylinder (10 mm length, $0.4 \mu \mathrm{m}$ diameter) to model the distal axon. A model MFB ( $4 \mu \mathrm{m}$ diameter) was placed in the middle of the cylinder. In $\boldsymbol{E}$, the eight-state model with proximal axonal kinetics was used. In $\boldsymbol{F}$, the E\&J MFB model with faster inactivation kinetics was used. The fast inactivation kinetics was required to obtain a low energy efficiency value during the short time course of the MFB AP.

Similarly, when the M\&S model was used to simulate Nav channels of layer 5 pyramidal cells, APs were initiated in the dendrites from $\sim 1 \mathrm{nS}$ per synapse on (Fig. $6 \mathrm{~B}$, continuous orange curve). This was not due to a difference in voltage dependence of gating models because similar results were observed when the voltage dependence of the M\&S model was shifted by $10 \mathrm{mV}$ to the left to match the peak activation curve of our eight-state gating model (supplemental Fig. 7, available at www.jneurosci.org as supplemental material). By contrast, when Nav channel density was increased to $2250 \mathrm{pS} \cdot \mu \mathrm{m}^{-2}$, similar to the values reported for the AIS in layer V pyramidal cells, the M\&S model was able to faithfully reproduce the robust axonal AP initiation in mossy fiber axons (Fig. $6 \mathrm{~B}$, dashed orange curve). Replacing the original eight-state model with the E\&J model throughout the axon led to dendritic AP initiation from $\sim 0.5 \mathrm{nS}$ per synapse on (Fig. $6 \mathrm{~B}$, continuous blue curve), suggesting that Nav channels in the proximal and the distal axon are specialized to serve distinct functions.

We next examined how the low density of Nav channels in the AIS would affect the impact of proximal axonal inhibition on AP output (Fig. 6C). Thirty inhibitory synaptic conductance changes were distributed along the proximal $30 \mu \mathrm{m}$ of the axon to simulate synaptic contacts between axoaxonic cells and granule cell axons (Buhl et al., 1994a). The conductance of the inhibitory synapses was increased between simulation runs while APs were evoked by near-threshold excitatory synapses with a constant conductance. When the eightstate model with fast axonal gating was used, AP generation was abolished at $\sim 1$ $\mathrm{nS}$ per inhibitory synapse (continuous black curve in Fig. 6C). By contrast, the inhibitory conductance had to be increased 1.7-fold when the M\&S model with a high axonal Nav channel density was used $\left(2250 \mathrm{pS} \cdot \mu \mathrm{m}^{-2}\right)$ (dashed orange curve in Fig. 6C).

Together, these results show that fast axonal activation kinetics is required to reproduce experimental findings obtained with low- and high-intensity synaptic stimulation (Schmidt-Hieber et al., 2008) with a relatively low density of Nav channels in the proximal mossy fiber axon. At the same time, the low density of Nav channels allows axoaxonic interneurons to efficiently modulate AP output.

\section{Nav channel gating promotes energy efficiency of AP initiation}

It has recently been suggested that AP generation in principal neurons is relatively energy efficient because of a small overlap of $\mathrm{Na}^{+}$and $\mathrm{K}^{+}$currents during the time course of the AP (Alle et al., 2009; Carter and Bean, 2009). In mossy fiber boutons, the $\mathrm{Na}^{+}$charge transfer during an AP has been reported to be 1.3 times the theoretical minimum that is required to depolarize the membrane (Alle et al., 2009). To assess the energy efficiency of AP initiation in the proximal mossy fiber axon, we simulated APs at room temperature in a long cylinder (Fig. 7; see supplemental material, available at www.jneurosci.org). Energy efficiency was quantified as the ratio of total $\mathrm{Na}^{+}$charge transfer during an AP and the theoretical minimal charge that is required to depolarize the cell membrane to the AP peak amplitude (Carter and Bean, 2009). When the experimentally determined inactivation kinetics was used, energy efficiency was 1.24 (Fig. $7 A$ ), similar to values that have recently been reported for the soma and axon of principal neurons (Alle et al., 2009; Carter and Bean, 2009). Simulations performed at physiological temperatures yielded similar results (supplemental Fig. 8, available at www.jneurosci.org as supplemental material). To determine the influence of Nav channel inactivation kinetics on energy efficiency, we varied inactivation rates by multiplying the original rates with a factor $f_{h}$ (Fig. $7 B-D$ ). These simulations revealed that the energy efficiency obtained with the experimentally determined inactivation kinetics (Fig. 7D, blue line) was very close to the minimal value of 1.21 (Fig. $7 D$, red line). Interestingly, energy efficiency worsened when inactivation kinetics was accelerated 
beyond $f_{h}>1.5$ because of fast recovery from inactivation (Fig. $7 C)$, suggesting that Nav channels are operating in an optimized kinetic range. Since we adjusted $\bar{g}_{\mathrm{K}}$ in each simulation individually so that the decay and half duration of the simulated AP closely matched experimentally determined values, Kv channel activation kinetics should only have little impact on our energy efficiency estimates (see supplemental Material for details). We tested this prediction by multiplying $\mathrm{Kv}$ channel activation rates with a factor of 0.5 (dotted-dashed curve in Fig. 7D) or 1.5 (dashed curve in Fig. 7D). Indeed, energy efficiency varied little for the different Kv channel kinetics. Most importantly, the energy efficiency obtained with the experimentally determined inactivation kinetics $\left(f_{h}=1\right)$ remained very close to the minimal value independent of the Kv channel activation kinetics.

Fast APs may decrease energy efficiency because of incomplete Nav channel inactivation (Carter and Bean, 2009). In MFBs in the distal granule cell axon, APs have a half-duration of $<1 \mathrm{~ms}$ (Bischofberger et al., 2002) (recorded at room temperature), but energy efficiency is close to the optimum (Alle et al., 2009). When we used our eight-state model from the proximal axon to replicate a fast $\mathrm{AP}$ in a compartmental model of an MFB, we failed to reproduce this finding (Fig. $7 E$ ). By contrast, when we used the E\&J model with $\sim 50 \%$ faster inactivation kinetics, energy efficiency was close to the values that have been found experimentally (Fig. 7F). Hence, inactivation kinetics in the proximal and distal axon appears to be adapted to the slow and rapid AP repolarization time courses, respectively. Our energy efficiency simulations show that the specialized kinetics of Nav channels in the initial segment of nonmyelinated axons allows initiating APs with little ion flow, thereby promoting energy efficiency. Together, low density and optimized gating kinetics of axonal Nav channels in hippocampal mossy fibers support robust AP initiation with minimal current flow.

\section{Discussion}

Our experimental data and simulations indicate that a moderately high Nav channel density and fast gating kinetics in the axon support robust and energy-efficient AP initiation in hippocampal mossy fibers. Nonmyelinated CNS axons such as hippocampal mossy fibers do not possess an anatomically defined axon initial segment or a first node of Ranvier. Nevertheless, our data show that a localized spike initiation zone is generated in the proximal part of the axon $(\sim 15-30 \mu \mathrm{m}$ from soma) by an enhanced Nav channel density ( $\sim 2$ to 9 times). In addition, we found that gating kinetics is up to twice as fast in the axon as in the granule cell soma, contributing to robust axonal AP initiation.

Comparing our data with Nav channel gating kinetics in MFBs (Engel and Jonas, 2005) suggests that Nav channel function is compartmentalized throughout the length of the mossy fiber axon. While fast activation kinetics in the proximal axon supports robust AP initiation, fast inactivation kinetics in more distal MFBs contributes to efficient $\mathrm{Na}^{+}$entry during fast APs (Fig. 8). This heterogeneous distribution of functional Nav channel properties raises the question of the underlying subunit composition. It has recently been shown that Nav 1.6 is the predominant $\alpha$-subunit in the proximal granule cell axon, while Nav 1.2 cannot be detected (Kress et al., 2010). In good agreement with this finding, channels composed of the Nav 1.6 subunit have been reported to exhibit more hyperpolarized peak activation and steady-state inactivation curves than Nav 1.2 channels (Rush et al., 2005). However, Nav 1.6 expression alone cannot account for the faster activation and inactivation kinetics in the axon (Rush et al., 2005), suggesting that auxiliary $\beta$-subunits

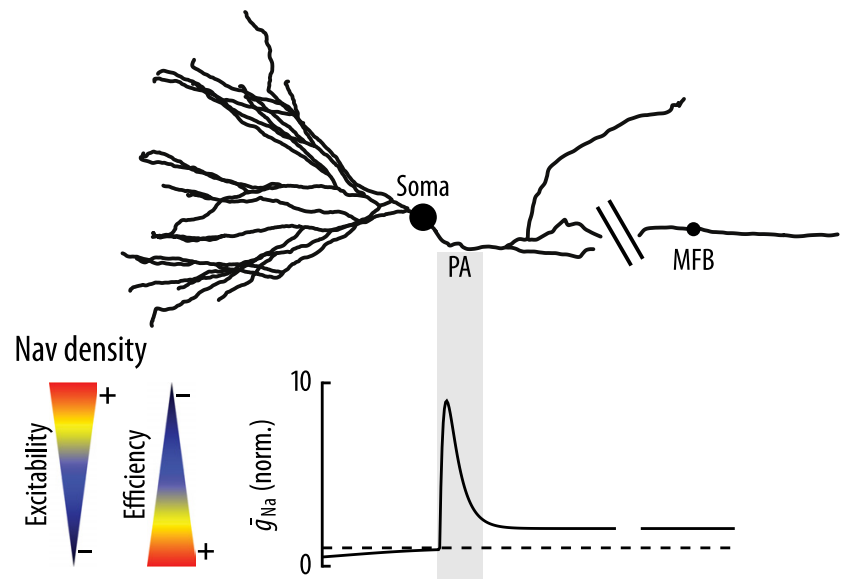

Activation
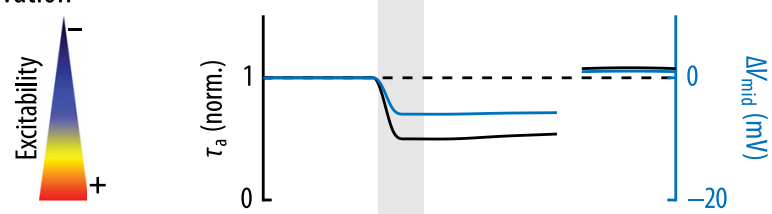

Inactivation
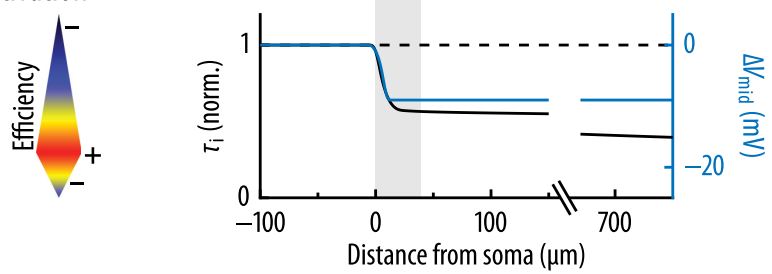

Figure 8. Specialized functional properties of Nav channels in subcellular compartments. The scheme summarizes the heterogeneous distribution of Nav channel density (top), activation kinetics (middle), and inactivation kinetics (bottom) in granule cells. $\mathrm{Na}^{+}$conductance density, inactivation, and activation time constants were normalized to the somatic values. Midpoint potentials are expressed as the difference with the somatic value. The heat maps on the left summarize the effects of the kinetic properties on excitability and energy efficiency (red, high excitability/good efficiency; blue, low excitability/bad efficiency). Data for the soma and proximal axon (PA) are from this study, and data for the MFB were taken from Engel and Jonas (2005).

might contribute to the kinetic differences. $\beta 1-3$ subunits have been reported to accelerate the inactivation time course when coexpressed with $\alpha$-subunits (Isom et al., 1992; Smith et al., 1998; Morgan et al., 2000). Of these, $\beta 2$ and $\beta 3$ are strongly expressed in the dentate gyrus (Morgan et al., 2000; Yu et al., 2003). Therefore, it seems likely that heteromers of Nav $1.6 \alpha$-subunits with a varying proportion of $\beta 2 / 3$ auxiliary subunits could explain the compartmentalized function of Nav channels along the mossy fiber axon. Since these axons have very thin diameters of $<500$ $\mathrm{nm}$, electron microscopy along with immunohistochemistry may be necessary to address this question.

Our results suggest that the mechanisms governing action potential initiation in thin nonmyelinated mossy fiber axons are substantially different from those in myelinated axons of cortical pyramidal cells. The peak Nav channel density in the axon appears to be smaller in granule cells $\left(\sim 1670 \mathrm{pS} \cdot \mu \mathrm{m}^{-2}\right.$ vs 2500 $\mathrm{pS} \cdot \mu \mathrm{m}^{-2}$ in pyramidal neurons) (Kole et al., 2008; Hu et al., 2009), and the spatial extent of this enhanced density differs markedly. In pyramidal cells, the Nav channel density appears to be high throughout the AIS until $\sim 50 \mu \mathrm{m}$ from the axon hillock. By contrast, in mossy fibers, the density decays within 10-35 $\mu \mathrm{m}$ from soma to values of $\sim 400 \mathrm{pS} \cdot \mu \mathrm{m}^{-2}$, leading to an average 
density of $\sim 1000 \mathrm{pS} \cdot \mu \mathrm{m}^{-2}$ within the proximal $35 \mu \mathrm{m}$ of the axon. Furthermore, the Nav channel densities measured in outside-out patches excised from the proximal axon are perfectly consistent with the properties of the AP waveform measured in whole-cell configuration (supplemental Fig. 6, available at www. jneurosci.org as supplemental material), indicating that axonal $\mathrm{Na}^{+}$currents can be reliably measured in outside-out patches (Hu et al., 2009).

On the other hand, somatic Nav channel density in granule cells appears to be higher than in layer V pyramidal cells $(\sim 200$ $\mathrm{pS} \cdot \mu \mathrm{m}^{-2}$ vs $\sim 80 \mathrm{pS} \cdot \mu \mathrm{m}^{-2}$ ) (Hu et al., 2009). One reason for this might be that the axon initial segment of layer $\mathrm{V}$ pyramidal cells has a substantially larger diameter $(\sim 1 \mu \mathrm{m}$ beyond $40 \mu \mathrm{m}$ distance from the soma) (Kole et al., 2008) than the proximal mossy fiber axon $(0.3-0.4 \mu \mathrm{m}$ beyond $\sim 30 \mu \mathrm{m}$ distance from the soma). Comparing compartmental models of a layer $\mathrm{V}$ pyramidal cell (Kole et al., 2008) and a granule cell (Schmidt-Hieber et al., 2007), we estimate that the surface area of the pyramidal cell axon initial segment is $\sim 6$ times larger than that of the proximal granule cell axon within $40 \mu \mathrm{m}$ distance from the soma. In combination with the higher Nav channel density, axial current flow from the axon into the soma during the AP onset is therefore expected to be substantially larger in pyramidal cells than in granule cells. It seems plausible that the higher Nav channel density in the granule cell soma compensates for the smaller axial current flow provided by the axon.

The higher Nav channel density in pyramidal cell axons might be related to the fact that the APs are not only initiated in the proximal part of the axon, but also have to be reliably propagated toward the first node of Ranvier via axial current flow. By contrast, in nonmyelinated axons of granule cells, axonal Nav channels in neighboring compartments actively contribute to continuous axonal AP propagation. Interestingly, the asymptotic value for axonal Nav channel density in our study $\left(\sim 400 \mathrm{pS} \cdot \mu \mathrm{m}^{-2}\right)$ (Fig. $1 E$ ) is very close to what was reported for Nav channels in mossy fiber boutons (490 $\mathrm{pS} \cdot \mu \mathrm{m}^{-2}$ ) (Engel and Jonas, 2005), indicating that the channel density might be homogeneously distributed along the distal mossy fiber axon. The relatively low Nav channel density in the proximal granule cell axon is also in good agreement with a lower PanNav immunostaining density as compared to CA3 pyramidal cell axons (Kress et al., 2010). When compared to a model of Nav channel gating in pyramidal cell axons, activation kinetics of axonal Nav channels is substantially faster in granule cells than in pyramidal cells (Fig. 6A). The faster gating appears to compensate for the lower density of Nav channels at the AP initiation site. As a consequence, AP threshold is always reached first in the axon, even during high background synaptic activity (Fig. 6B).

It has recently been suggested that $\mathrm{Nav}$ and $\mathrm{Kv}$ channel gating in MFBs is optimized for energy-efficient charging of the axonal membrane (Alle et al., 2009). Here we show that inactivation kinetics of Nav channels in the proximal mossy fiber axon is tuned for efficient depolarization of the membrane during AP initiation, resulting in efficient current flow close to the theoretical minimum. It remains to be determined whether these findings generalize to unmyelinated proximal axons of other cell types. The proximal part of cortical myelinated axons and nonmyelinated mossy fibers is preferentially targeted by axoaxonic cells (Han et al., 1993; Buhl et al., 1994a,b). If APs are initiated with minimal charge, this population of GABAergic interneurons can powerfully modulate the timing and rate of spike output by relatively small conductance changes.

The results show that a moderately higher density of Nav channels with specialized gating properties provide a reliable and unique spike initiation zone in the proximal mossy fiber axon in the absence of a morphologically defined axon initial segment. As a consequence, synaptic inputs from thousands of different dendritic locations are propagated toward the soma and evaluated in a remarkably energy-efficient manner at a single point in the axon close to the axon hillock.

Note added in proof. Recently, another study investigated $\mathrm{Na}^{+}$ influx in axons of neocortical pyramidal cells using $\mathrm{Na}^{+}$sensitive fluorescent imaging (Fleidervish et al., 2010). The authors find that Nav channel density in initial segments of these myelinated axons might be $\sim 3$ times the somatic density, similar to our estimates for unmyelinated axons.

\section{References}

Alle H, Roth A, Geiger JRP (2009) Energy-efficient action potentials in hippocampal mossy fibers. Science 325:1405-1408.

Attwell D, Gibb A (2005) Neuroenergetics and the kinetic design of excitatory synapses. Nat Rev Neurosci 6:841-849.

Attwell D, Laughlin SB (2001) An energy budget for signaling in the grey matter of the brain. J Cereb Blood Flow Metab 21:1133-1145.

Baranauskas G, Martina M (2006) Sodium currents activate without a Hodgkin-and-Huxley-type delay in central mammalian neurons. J Neurosci 26:671-684.

Bischofberger J, Geiger JRP, Jonas P (2002) Timing and efficacy of $\mathrm{Ca}^{2+}$ channel activation in hippocampal mossy fiber boutons. J Neurosci 22:10593-10602.

Buhl EH, Halasy K, Somogyi P (1994a) Diverse sources of hippocampal unitary inhibitory postsynaptic potentials and the number of synaptic release sites. Nature 368:823-828.

Buhl EH, Han ZS, Lörinczi Z, Stezhka VV, Karnup SV, Somogyi P (1994b) Physiological properties of anatomically identified axo-axonic cells in the rat hippocampus. J Neurophysiol 71:1289-1307.

Carnevale NT, Hines ML (2006) The NEURON book. Cambridge, NY: Cambridge UP.

Carter BC, Bean BP (2009) Sodium entry during action potentials of mammalian neurons: incomplete inactivation and reduced metabolic efficiency in fast-spiking neurons. Neuron 64:898-909.

Chanda B, Asamoah OK, Bezanilla F (2004) Coupling interactions between voltage sensors of the sodium channel as revealed by site-specific measurements. J Gen Physiol 123:217-230.

Colbert CM, Pan E (2002) Ion channel properties underlying axonal action potential initiation in pyramidal neurons. Nat Neurosci 5:533-538.

Colquhoun D, Hawkes AG (1977) Relaxation and fluctuations of membrane currents that flow through drug-operated channels. Proc R Soc Lond B Biol Sci 199:231-262.

Engel D, Jonas P (2005) Presynaptic action potential amplification by voltage-gated $\mathrm{Na}^{+}$channels in hippocampal mossy fiber boutons. Neuron 45:405-417.

Fleidervish IA, Lasser-Ross N, Gutnick MJ, Ross WN (2010) $\mathrm{Na}^{+}$imaging reveals little difference in action potential-evoked $\mathrm{Na}^{+}$influx between axon and soma. Nat Neurosci 13:852-860.

Geiger JRP, Bischofberger J, Vida I, Fröbe U, Pfitzinger S, Weber HJ, Haverkampf K, Jonas P (2002) Patch-clamp recording in brain slices with improved slicer technology. Pflügers Arch 443:491-501.

Han ZS, Buhl EH, Lörinczi Z, Somogyi P (1993) A high degree of spatial selectivity in the axonal and dendritic domains of physiologically identified local-circuit neurons in the dentate gyrus of the rat hippocampus. Eur J Neurosci 5:395-410.

Hodgkin AL, Huxley AF (1952) A quantitative description of membrane current and its application to conduction and excitation in nerve. J Physiol 117:500-544.

Hu W, Tian C, Li T, Yang M, Hou H, Shu Y (2009) Distinct contributions of $\mathrm{Na}_{\mathrm{v}} 1.6$ and $\mathrm{Na}_{\mathrm{v}} 1.2$ in action potential initiation and backpropagation. Nat Neurosci 12:996-1002.

Isom LL, De Jongh KS, Patton DE, Reber BFX, Offord J, Charbonneau H, Walsh K, Goldin AL, Catterall WA (1992) Primary structure and functional expression of the $\beta 1$ subunit of the rat brain sodium channel. Science 256:839-842.

Keynes RD, Rojas E (1976) The temporal and steady-state relationships be- 
tween activation of the sodium conductance and movement of the gating particles in the squid giant axon. J Physiol 255:157-189.

Kole MHP, Letzkus JJ, Stuart GJ (2007) Axon initial segment Kv1 channels control axonal action potential waveform and synaptic efficacy. Neuron 55:633-647.

Kole MHP, Ilschner SU, Kampa BM, Williams SR, Ruben PC, Stuart GJ (2008) Action potential generation requires a high sodium channel density in the axon initial segment. Nat Neurosci 11:178-186.

Kress GJ, Dowling MJ, Meeks JP, Mennerick S (2008) High threshold, proximal initiation, and slow conduction velocity of action potentials in dentate granule neuron mossy fibers. J Neurophysiol 100:281-291.

Kress GJ, Dowling MJ, Eisenman LN, Mennerick S (2010) Axonal sodium channel distribution shapes the depolarized action potential threshold of dentate granule neurons. Hippocampus 20:558-571.

Lorincz A, Nusser Z (2010) Molecular identity of dendritic voltage-gated sodium channels. Science 328:906-909.

Mainen ZF, Joerges J, Huguenard JR, Sejnowski TJ (1995) A model of spike initiation in neocortical pyramidal neurons. Neuron 15:1427-1439.

Martina M, Jonas P (1997) Functional differences in $\mathrm{Na}^{+}$channel gating between fast-spiking interneurones and principal neurones of rat hippocampus. J Physiol 505:593-603.

Morgan K, Stevens EB, Shah B, Cox PJ, Dixon AK, Lee K, Pinnock RD, Hughes J, Richardson PJ, Mizuguchi K, Jackson AP (2000) $\beta 3$ : an additional auxiliary subunit of the voltage-sensitive sodium channel that modulates channel gating with distinct kinetics. Proc Natl Acad Sci U S A 97:2308-2313.

Oxford GS (1981) Some kinetic and steady-state properties of sodium channels after removal of inactivation. J Gen Physiol 77:1-22.

Rush AM, Dib-Hajj SD, Waxman SG (2005) Electrophysiological proper- ties of two axonal sodium channels, Nav 1.2 and Nav 1.6, expressed in mouse spinal sensory neurones. J Physiol 564:803-815.

Sakmann B, Neher E (1995) Geometric parameters of pipettes and membrane patches. In: Single-channel recording (Sakmann B, Neher E, eds), pp 637-650. New York: Plenum.

Schmidt-Hieber C, Jonas P, Bischofberger J (2007) Subthreshold dendritic signal processing and coincidence detection in dentate gyrus granule cells. J Neurosci 27:8430-8441.

Schmidt-Hieber C, Jonas P, Bischofberger J (2008) Action potential initiation and propagation in hippocampal mossy fibre axons. J Physiol 586:1849-1857.

Shu Y, Hasenstaub A, Duque A, Yu Y, McCormick DA (2006) Modulation of intracortical synaptic potentials by presynaptic somatic membrane potential. Nature 441:761-765.

Smith MR, Smith RD, Plummer NW, Meisler MH, Goldin AL (1998) Functional analysis of the mouse Scn8a sodium channel. J Neurosci 18:6093-6102.

Stuart G, Spruston N, Sakmann B, Häusser M (1997) Action potential initiation and backpropagation in neurons of the mammalian CNS. Trends Neurosci 20:125-131.

Vandenberg CA, Bezanilla F (1991) A sodium channel gating model based on single channel, macroscopic ionic, and gating currents in the squid giant axon. Biophys J 60:1511-1533.

Yu FH, Westenbroek RE, Silos-Santiago I, McCormick KA, Lawson D, Ge P, Ferriera H, Lilly J, DiStefano PS, Catterall WA, Scheuer T, Curtis R (2003) Sodium channel $\beta 4$, a new disulfide-linked auxiliary subunit with similarity to $\beta 2$. J Neurosci 23:7577-7585. 